\title{
Modelo para la gestión de la innovación en las micro, pequeñas y medianas empresas (mipymes) en Colombia
}

\author{
Jose Eisenover Cely Rojas
}

Universidad Pedagógica y Tecnológica de Colombia momercely@gmail.com.

\begin{abstract}
Resumen
Este trabajo se presenta como resultado del proceso investigativo que parte de las inquietudes generadas por los hallazgos de la primera investigación de la Red de Estudios sobre Innovación, adelantado en concurso por diez de las principales universidades colombianas y el Observatorio Colombiano de Ciencia y Tecnología (OCYT), el cual puntualiza que las Mipymes colombianas presentan escasos e insipiente resultados de innovación, en la mayoría de los casos (98\%), las empresas carecen de conocimiento y recursos para su desarrollo, así como de una estructura organizacional y relaciones de apoyo que soporten este tipo de ejercicios; en consecuencia esta situación incide en gran medida en las posibilidades de perdurabilidady capacidad competitiva de este tipo de organizaciones.
\end{abstract}

La propuesta de modelo de gestión de la innovación en Mipymes de Colombia, que aqui se presenta, denominado como "Modelo de la Triada", es el resultado innedito del proceso investigativo adelantado en la tesis doctoral titulada "Propuesta de modelo de incorporación y formalización de procesos de desarrollo de productos en las Mipymes manufactureras del sector plásticos del departamento de Boyacá (Colombia), como aporte al fortalecimiento de la competitividad de estas organizaciones"Cely, J.(2017). Este modelo representa y describe el sistema de factores tanto internos como externos que definen la realidad de las empresas y que por sus características determinan las posibilidades de desarrollar procesos de innovación.

El modelo busca explicar y caracterizar los condicionantes a tener en cuenta para promover la innovación en las organizaciones, propone una estructura de estudio y análisis formal que permite establecer las bases para el diagnóstico de problemas relacionados con la gestión de la innovación, útil para la toma de decisiones, la configuración de planes y estrategias para la gestión a este nivel. 
Modelo para la gestión de la innovación en las micro, pequeñas y medianas empresas (Mipymes) en Colombia

El modelo distingue cuatro grupos generales de Factores: Factores claves o de primer nivel, factores relevantes o de segundo nivel, factores determinantes o de tercer nivel y factores contextuales; que inician estableciéndose en el ámbito externo de las empresas y que repercuten en la configuración de factores del entorno interno de las organizaciones.

Palabras claves: innovación, gestión, modelo, Mipymes.

\section{Introdución}

De acuerdo con los indicadores de competitividad internacional, como lo son el Índice de Competitividad Global, desarrollado por el Foro Económico Mundial (FEM), el Índice de Competitividad del Institute for Management Development (IMD) y el Índice de Facilidad para Hacer Negocios (Doing Business, DB) del Banco Mundial, Colombia se ubica por debajo de la media der ranking que para este caso se establece. Estas mediciones sobre el desempeño en términos de competitividad de los países, contienen indicadores de índole macroeconómica y microeconómico, que relacionan aspectos como: las características del gobierno, los planes de desarrollo y su ejecución, al igual que la capacidad de integración de las empresas en sus diferentes sectores, el desarrollo de productos, la capacidad de innovación de estas y su flexibilidad como atributo para responder a la dinámica de cambio de la economía global, han puesto de manifiesto la debilidad pero también el reto que Colombia tiene para mejorar en estos aspectos. La principal debilidad que el país presenta, se esquematiza sobre los elementos que determinan las posibilidades de desarrollo de procesos de innovación en las empresas.

En Colombia se tipifican las Mipymes de acuerdo con los activos totales y el número de empleados que se tienen (Ley 905 de 2004). Es así como se cataloga una empresa mediana a aquellas organizaciones que tiene entre cincuenta y uno (51) y doscientos empleados (200) y sus activos totales corresponden entre 5.001 y 15.000 salarios mínimos mensuales (salario mínimo mensual: $\$ 737.717$ pesos, $€ 246$ aproximadamente), mientras que una empresa es catalogada como pequeña cuando cuenta con un número de empleados de entre once (11) y cincuenta (50) y sus activos totales corresponden entre 501 y 5.000 salarios mínimos mensuales. En estas condiciones, las Mipymes son el 94,7\% en el país, aportando un $66 \%$ del empleo industrial, desarrollan el $25 \%$ de las exportaciones no convencionales y pagan el 50\% de los salarios, según la información suministrada el Ministerio de Desarrollo (2015). Las Mipymes en Colombia según estos mismos datos, representan casi una tercera parte de la producción nacional, participan con un $57 \%$ en la generación de empleo industrial, siendo estas las que definen en gran medida la dinámica económica nacional. 
En estas condiciones, el presente trabajo propone un modelo gestión de la innovación para las Mipymes, que busca en primer término, establecer una estructura de análisis para la caracterización de los factores que determinan las posibilidades de incorporación de procesos de innovación en estas organizaciones y con ello facilitar su análisis y promover la toma de decisiones al respecto de la definición de planes y estrategias para la gestión de la innovación.

\section{Marco de Referencia}

De acuerdo a los resultados de la primera investigación de la Red de Estudios sobre Innovación, adelantado en concurso por diez de las principales universidades colombianas y el Observatorio Colombiano de Ciencia y Tecnología (OCYT), los cuales se publicaron en el libro La Innovación Tecnológica en la Industria Colombiana (Malaver, Vargas y Zerda, 2012), las Mipymes colombianas presentan un escasa e insipiente formalización de sus procesos de innovación, en la mayoría de los casos (98\%), las empresas carecen de una dependencia formal encargada de gestionarla y desarrollarla; el carácter de los procesos de gestión de la innovación es abiertamente informal, lo cual incide en gran medida en los rasgos que perfilan sus innovaciones y la capacidad de establecer verdaderas ventajas competitivas.

Según este estudio, la innovación en la mayoría de los casos no es una práctica permanente y sistemática, pues no se incorporan estructuralmente en estas organizaciones unidades o personas especializadas encargadas para adelantar esta actividad de manera explícita, por lo general se adelantada por el empresario, gerente o por la dirección de producción, en ausencia de ejercicios cienciométricos o de prospectiva tecnológica o de revisión de bases de patentes o de participación en proyectos de investigación tecnológica; de esta manera el origen de los nuevos productos por ejemplo, se da por el mejoramiento de uno previo, impulsado por las ideas captadas por la asistencia a ferias, consulta de revistas especializadas o en redes electrónicas y en algunos casos por los requerimientos directos de los clientes. En consecuencia los nuevos productos de las Mipymes colombianas son el resultado de la mejora o reformulación de uno ya existente y su origen proviene de fuentes internas antes que externas (OECD, 1996, y Jaramillo, Lugones, y Salazar, 2000).

\section{Metodología}

El estudio es de tipo descriptivo analítico, con enfoque cuantitativo, su diseño es de carácter no experimental que en principio se presenta exploratorio por la ausencia de estudios previos, utiliza como instrumento de recolección de información la encuesta descriptiva, la cual estuvo dirigida a los directivos de las organizaciones y fue aplicada mediante un muestreo aleatorio simple a un grupo de 356 empresas, subdivididas por los 
principales sectores económicos del país, tales como: Plásticos, metalmecánico, maderero, alimentos, construcción, y servicios, entre otros. De otra parte, el instrumento fue estructurado a partir de tres secciones, que a través de 43 preguntas cerradas de selección múltiple y un análisis correlacional permitió indagar y establecer la relación entre los siguientes aspectos o variables:

Variables internas: Orientación y organización de la empresa, percepción de la innovación, liderazgo de la dirección, recursos para la innovación, medio de adquisición y manejo de información y conocimiento utilizados por la empresa; implementación de las TIC; herramientas o instrumentos que utiliza la empresa en cada una de las fases del proceso de desarrollo de productos; equipos, software y hardware especializados que utiliza la empresa para el desarrollo de productos.

Adicionalmente y definidas como variables externas, se realizó un estudio documental que abordo las variables: Sistema nacional de ciencia y tecnología, la normatividad y legislación aplicable a los procesos y gestión de la innovación, entorno de la protección de la propiedad industrial, redes de cooperación y apoyo para la innovación y el estado de los sectores económicos.

Para el procesamiento de los datos se utilizó como herramienta el programa estadístico informático SPSS y la técnica de análisis multivariado.

Se utilizaron como fuentes de información secundaria, documentos desarrollados por diferentes organizaciones como: El Departamento Administrativo Nacional de Estadística DANE, Ministerio de Industria Comercio y Turismo - MinCIT , La Asociación Nacional de Instituciones Financieras ANIF, Ministerio de las Tecnologías de la Información y las Comunicaciones MINTIC, entre otras, que de una u otra manera han estudiado a nivel nacional o regional, aspectos y factores relacionados en este proyecto.

\section{Resultados}

A continuación, se presenta a modo de resultados de investigación, la propuesta de modelo de gestión de la innovación en las micro, pequeñas y medianas empresas (Mipymes) de Colombia como aporte al fortalecimiento de la competitividad de estas organizaciones. En este modelo se representa y describe el sistema de factores que hacen parte de la realidad de las empresas referidas y que inciden en la incorporación y formalización de los procesos de innovación.

El modelo recurre a la representación gráfica, para expresar el conocimiento construido a través del análisis de la teoría e información recolectada acerca del entorno de los sectores manufactureros más representativos de la economía colombiana y de las características de las organizaciones destacadas en estos sectores, en lo que tiene que ver a los procesos de innovación. Este modelo permite la comprensión de la complejidad de la realidad y 
condiciones que al respecto de la gestión de la innovación se presenta en las Mipymes, identificando las relaciones que se dan entre los factores que hacen parte en la construcción de la mencionada realidad. En este sentido el modelo que se define como "De la Triada", es el resultado de la modelación del objeto de conocimiento abordado en este proceso investigativo, se presenta como un ejercicio de construcción mental que representa el conocimiento construido de la observación y análisis.

\subsection{Objetivos del modelo}

La propuesta de modelo busca:

- Explicar y caracterizar las condiciones a tener en cuenta para promover la formalización e incorporación de proceso de innovación en las Mipymes manufactureras.

- Proponer una estructura, de estudio y análisis formal de la gestión de los procesos de innovación en las Mipymes.

- Establecer una base para el diagnóstico de problemas relacionados con la gestión de la innovación tanto a nivel interno de las organizaciones, como de los sectores.

- Proponer parámetros que orienten la toma de decisiones al respecto de la definición de planes y estrategias para la gestión de la innovación en el entorno particular de las Mipymes.

\subsection{Principios y delimitación del modelo}

La construcción del modelo para la formalización e incorporación de procesos de innovación al interior de las Mipymes manufactureras en Colombia parte de los siguientes principios:

- El proceso de innovación es complejo en la medida que cuenta con múltiples agentes que lo influencian y condicionan, demandando igualmente múltiples competencias, cualidades y características de la organización, Rosegger (1986).

- El modelo asume la realidad del proceso de innovación desde la perspectiva de la gestión, sin profundizar en los aspectos prácticos operativos del mismo, por cuanto estos no son el motivante para la investigación. En consecuencia, el modelo expresa orientaciones para la gestión los procesos de innovación, al interior de las Mipymes.

- Como efecto de lo anterior, los términos incorporación y formalización deben ser entendidos como los define la Real Academia de la Lengua Española: 
- Incorporar: Del latín incorporare. Verbo que expresa la acción, proceso, estados o actitud de unir una cosa a otra para que haga un todo con ella.

Formalizar: Verbo que expresa la acción, proceso, estado o actitud de revestir algo de los requisitos legales o de procedimiento, dar estructura formal a una proposición, concretar, precisar algo, dar carácter de seriedad a algo que no lo tenía, representar algo como ideas informaciones o conocimientos, con los recursos formales de un sistema.

- Estas definiciones son las orientadoras del ejercicio investigativo, permitiendo relacionar los factores tanto internos como externos que inciden en las Mipymes colombianas, como elementos que deben ser asimilados y garantizados por estas organizaciones para permitir la gestión de los procesos de desarrollo de productos.

- $\quad$ El modelo identifica los factores relevantes para la incorporación y formalización de procesos de desarrollo de productos, para lo cual se catalogan como: Factores determinantes o de primer nivel, Factores relevantes o de segundo nivel, factores claves o de tercer nivel. De igual manera se establecen las relaciones que se dan entre cada uno de ellos y el entorno al cual pertenecen.

- Tomando como base los resultados de los estudios realizados por Lugones (2007), al respecto de la identificación de los factores que condicionan el desarrollo de la innovación en las empresas y de acuerdo a las características de las Mipymes, los factores que se involucran en la propuesta de modelo Son:

\subsubsection{Factores externos}

Son los factores sobre los cuales se desenvuelven las organizaciones y determinan el ambiente y contexto de desarrollo.

- El estado del sector: En lo que tiene que ver con el desempeño del sector y el estado de competitividad del mismo.

- El entorno del desarrollo de la ciencia, la tecnología y la innovación: En lo particular de la existencia de políticas públicas de apoyo a la innovación tecnológica, especialmente a las pymes, cooperación en $\mathrm{I}+\mathrm{D}$, la existencia de redes de servicios tecnológicos, establecimiento de redes de cooperación con centros de investigación y universidades y el sistema nacional de educación.

- El estado de la Normatividad y Legislación: Aplicable a los procesos y gestión de la innovación, sistema de normas para los procesos de innovación, sistema de protección de la propiedad industrial.

- $\quad$ Entorno de la protección de la propiedad industrial.

- Redes de cooperación y apoyo para la innovación: Factor que se desarrolla dentro de la dinámica y entorno del desarrollo de la ciencia, la tecnología y la innovación; 
busca identificar las diferentes posibilidades de cooperación y apoyo, al igual que los mecanismos para acceder a ello.

\subsubsection{Factores internos}

Relaciona los aspectos que caracterizan la organización y son del entorno particular:

- La orientación de la empresa: Al mercado, a la producción, al costo, al valor o a la innovación.

- La percepción que se tiene acerca de la innovación al interior de la organización: La conceptualización y la importancia que se le da a la innovación al interior de la organización. La gestión del conocimiento al interior de la empresa.

- Los mecanismos de gestión del conocimiento: Al respecto de equipos de trabajo, las redes de colaboración que se establezcan dentro de la organización. las redes de colaboración entre organizaciones que compartan objetivos comunes, sistemas informáticos que permitan la creación y el mantenimiento de repositorios de conocimiento, las tecnologías de la información y comunicación (TIC) implementadas.

- La transferencia tecnológica: En lo que tiene que ver con las fuentes y mecanismos que utiliza la empresa.

- Los procesos de planeación para la gestión del proceso de innovación y la definición de plan estratégico.

- La organización para los procesos de innovación: Estructura organizativa, departamento y área funcional.

- Los procesos de dirección para la innovación: Responsabilidad de proyectos, compromiso y participación de la dirección, área y personal encargada de liderar los procesos de innovación, equipos de trabajo para los procesos de innovación, niveles de autonomía en la toma de decisiones.

- Los mecanismos de control para los procesos de innovación: implementados en la organización para la gestión de la innovación.

- Los recursos disponibles para asumir proceso de innovación: Financieros, físicos y humanos.

En consecuencia, el modelo de gestión de procesos de innovación, representa gráficamente en un plano abstracto y simplificado, los factores anteriormente mencionados, los estudia, los identifica y caracteriza, descubriendo sus relaciones al igual que sus cualidades, 
Modelo para la gestión de la innovación en las micro, pequeñas y medianas empresas (Mipymes) en Colombia

permitiendo entender y dar explicación a las proposiciones formuladas como hipótesis para esta investigación.

\subsection{Representación Gráfica del modelo de la triada para la gestión de la innovación en las Mipymes en Colombia}

A continuación en la figura $\mathrm{N}^{\circ} 1$, se representa gráficamente el modelo de gestión de la innovación para las Mipymes en Colombia, denominado como "Modelo de la Triada", el cual se construye sobre la relación de los factores que inciden en la gestión de innovación en estas organizaciones.

Figura $\mathrm{N}^{\circ}$ 1. Modelo de la Triada, para la Gestión de la Innovación en Mipymes

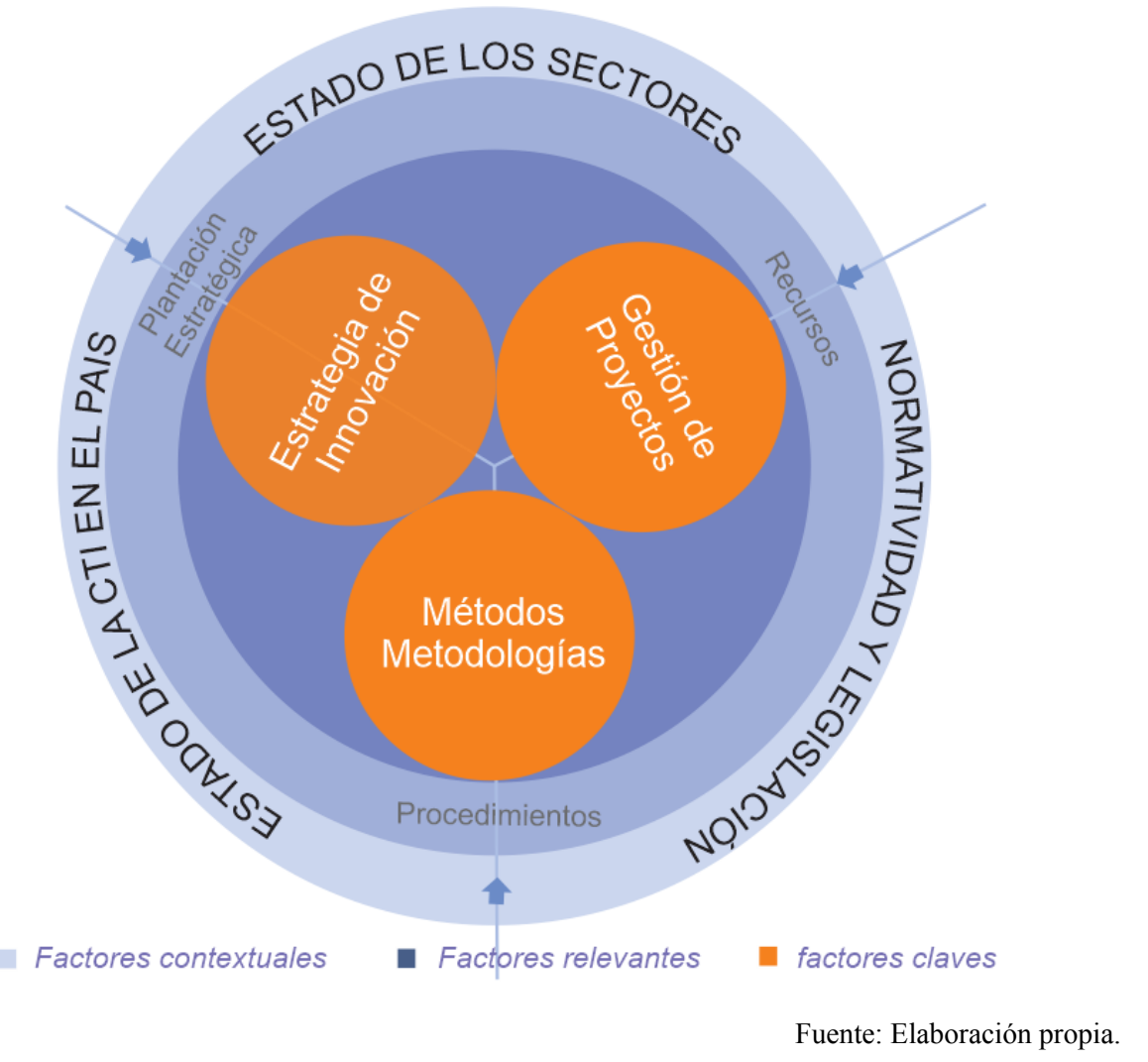




\subsection{Configuración del modelo}

El modelo establece los factores que inciden en la gestión de los procesos de innovación, se construye a través de tres niveles de jerarquía, apropiando el concepto "convergencia" concepto que se utiliza para describir la relación que tienen los factores estudiados, estableciendo la confluencia de estos en el propósito de incorporación y formalización de los procesos de innovación en las Mipymes en Colombia. De esta manera se puede identificar la incidencia de los factores relacionados, al igual que su procedencia.

Con esta característica del modelo, se establecen los siguientes elementos:

1. Clases de factores según su incidencia.

2. Entorno de procedencia de los factores.

\subsection{Elementos del modelo}

\subsubsection{El entorno de procedencia}

Hace referencia al contexto de donde se originan los factores, pudiéndose identificar:

- Un entorno interno, correspondiente al ambiente que constituye la propia empresa y sobre el cual se desarrollan la gestión de la misma, en este se contienen los factores claves y relevantes para la gestión de la innovación.

- El entorno externo, que se constituye con el macro ambiente o microambiente en el cual se desenvuelven las organizaciones. En este entorno se establecen los factores determinantes para la gestión de la innovación en las empresas, al igual que los factores denominados como contextuales.

En este aspecto, se puede identificar una dinámica de dependencia entre estos dos entornos, en la cual el entorno externo propicia las causas que determina las acciones del entorno interno, como mecanismo de reacción y respuesta a las condiciones que determina el macro y micro ambiente de las organizaciones.

\subsubsection{Factores según su incidencia}

A continuación de definen los factores que componen el modelo y de igual manera se identifican las relaciones que se dan entre estos de acuerdo también a sus entornos de procedencia. 


\subsubsection{Factores claves o de primer nivel}

Corresponde a los factores que por su incidencia en la incorporación y formalización de procesos de desarrollo de productos se consideran esenciales y básicos, e imprescindibles para la consecución del mencionado propósito. A este nivel se identifica que las organizaciones deben contar con: Una estrategia producto-mercado, una unidad de gestión de proyectos y procedimientos para el desarrollo de productos.

- Plan estratégico: Resultado del ejercicio de planeación estratégica y como respuesta a las amenazas y oportunidades presentes en el sector. La estrategia formulada debe estar orientada a promover el desarrollo y crecimiento de la empresa, por lo que adoptar una orientación a la innovación y al mercado, se constituye en un pilar fundamental. La estrategia debe en consecuencia estar claramente definida en términos de las expectativas de diferenciación y de desarrollo de la innovación.

- Procedimientos: Corresponde a la manera como la organización adelanta el proceso de innovación, la cual se establece a través de un método y una metodología en las que defina claramente las fase y actividades así como las herramientas e instrumentos que se utilizan para evolucionar el proceso. Al respecto se identifica la necesidad de incorporar métodos y metodologías que logren promover el desarrollo de la innovación.

- Unidad de gestión de proyectos: Como mecanismo para facilitar la adopción de los procesos de innovación en la estructura organizativa de la empresa. Su propósito es el de ejecutar los proyectos de innovación, establecidos en el plan estratégico. La unidad de gestión de proyectos, tiene la labor de promover y liderar el planteamiento y ejecución de los proyectos, ocuparse de la gestión de los recursos necesarios tales como financieros, humanos, tecnológicos y conocimiento, así como de la dirección administración, organización y control tanto de los recursos como de los proyectos, garantizándose la incorporación de los procesos de innovación en la organización.

Como aspecto importante, se puede establecer que estos tres factores, actúan de manera sinérgica, guardando una relación de mutua dependencia.

\subsubsection{Factores Relevantes o de segundo nivel}

Toman esta denominación, los factores que resultan ser significativos e importantes para la incorporación y formalización de los procesos de innovación, ya que estos se constituyen en los causales de la configuración de los factores claves o primarios, manteniendo una relación directa de dependencia hacia estos. Bajo esta denominación se distinguen: La orientación al mercado y a la ecología industrial de la empresa y la planeación estratégica que la organización debe realizar, los métodos y metodologías para el desarrollo de 
proyectos de innovación, la gestión del conocimiento y la transferencia tecnológica al igual que la planeación, dirección, organización y control de los procesos de desarrollo de productos.

A este grupo de factores pertenecen los recursos, representados por los financieros, talento humano y tecnológico requerido para el adelanto de procesos de innovación.

\subsubsection{Factores determinantes o de tercer nivel}

Con el mismo sentido de los factores relevantes, estos se constituyen en los causales de los ya mencionados. Estos factores fijan los términos y distinguen la procedencia así como el valor y sentido de los factores relevantes y en consecuencia de los claves, las relaciones que se dan sobre ellos, se establecieron con la definición de los factores que los preceden. Estos factores pertenecen al macro y micro ambiente de los diferentes sectores económicos y se establecen como: Redes de apoyo privadas y gubernamentales, condiciones y estado de la competitividad del sector, Normatividad y legislación aplicable a los procesos de innovación.

\subsubsection{Factores contextuales}

Corresponde a los factores del microambiente del sector, que determinan y condicionan las características del conjunto de los factores anteriormente relacionados.

A este nivel se distinguen: Política de Educación Nacional para la Competitividad, Política Nacional de Ciencia y Tecnología, Planes de desarrollo nacional y departamental, estado del sector plástico, Política Nacional de Producción y Consumo Limpio, Normas aplicables a envases y empaques plásticos, Normas para la gestión de la calidad, Normas para la gestión medioambiental, normas aplicables a la gestión de la investigación, desarrollo tecnológico e innovación.

\section{Conclusiones}

El presente modelo pretende aportar en la superación de las dificultades que a este nivel presentan las Mipymes, proporcionando una estructura, de estudio y análisis formal de la gestión del proceso de innovación, que permite explicar y caracterizar las condiciones y factores tanto internos como externos que inciden en su apropiación por parte de estas organizaciones y que son propuestos en el modelo y con ello propiciar parámetros que orienten la toma de decisiones al respecto de la definición de planes y estrategias para la gestión de la innovación en el entorno particular de las Mipymes, así como de las instituciones públicas y privadas interesadas en fortalecer las capacidades competitivas de los diferentes sectores de la economía colombiana. 
Modelo para la gestión de la innovación en las micro, pequeñas y medianas empresas (Mipymes) en Colombia

\section{Referencias}

Cely, J. (2017). Propuesta de modelo de incorporación y formalización de procesos de desarrollo de productos en las Mipymes manufactureras del sector plásticos del departamento de Boyacá (Colombia), como aporte al fortalecimiento de la competitividad de estas organizaciones. (Tesis doctoral innedita, Universidad Antonio de Nebrija, Madrid/España). Recuperado de www.turnitin.com

Jaramillo, H. L., \& F Salazar, M. (2000). Normalización de indicadores de innovación tecnológica en América Latina y el Caribe: manual de Bogotá (No. Doc. 21557) CO-BAC, Bogotá).

Jurado, J. M. V., Gracia, A. G., De Lucio, I. F., Conocimiento, I., \& Henríquez, L. A. M. (2005). Los determinantes de la innovación tecnológica en la empresa: una aproximación a través del concepto de capacidad de absorción.

Katz, R., \& Allen, T. J. (1985). Organizational issues in the introduction of new technologies. The management of productivity and technology in manufacturing, 2, 275-300.

Kao, J. (2007). Innovation nation: How America is losing its innovation edge, why it matters, and what we can do to get it back. Simon and Schuster.

Lugones, G., Suárez, D., \& Le Clech, N. (2007). Conducta innovativa y desempeño empresarial. Documentos de Trabajo de REDES N, 33.

Malaver, F., Vargas, M., \& Zerda, A. (2012). La innovación en la industria manufacturera colombiana: algunos avances y muchos desafíos. Vargas, $\mathrm{M}$.

Malaver Rodríguez, F., \& Vargas, M. (2004). El comportamiento innovador en la industria colombiana: una exploración de sus recientes cambios. Cuadernos de administración, 17(27).

Martínez, E. C., de Lucio, I. F., Marín, M. P., \& Boado, F. C. (2008). La transferencia de conocimientos desde las Humanidades: posibilidades y características. Arbor, 184(732), 619-636.

Manufacturera, E. A. (2002). Departamento Administrativo Nacional de EstadísticasDANE.

Martínez Villaverde, L., \& Villaverde, L. M. (2006). Gestión del cambio y la innovación en la empresa: Un modelo para la innovación empresarial (No. Sirsi) i9788498390087).

Montejo, M. J., \& Bravo, A. (2010). La innovación en sentido amplio: un modelo empresarial: análisis conceptual y empírico. Cotec.

OCDE, C. (2014). CEPAL,(2014). Perspectivas económicas de América Latina.

Observatorio Colombia de Ciencia y Tecnología. (2012). Plan estratégico departamental de Ciencia, Tecnología e Innovación - PEDCTI. Boyacá 2022: La ciencia, la tecnología y la innovación al servicio del desarrollo regional. Colombia: Ediciones Antropos Ltda., 304 p. Disponible en: http://www.dapboyaca.gov.co/wp-content/uploads/2014/ 03/PECDTIBoyaca.pdf

Rosegger, G. (1991). Advances in information technology and the innovation strategies of firms. Prometheus, 9(1), 5-20.

Rosegger, G. (1986). The Economics of Production \& Innovation, 2ed. Pergamon Press: Oxford, pp. 190-19.

Rosenberg, N., \& Frischtak, C. R. (1984). Technological innovation and long waves. Cambridge Journal of Economics, 8(1), 7-24. 